\title{
Diagnostic Value of Real-Time Ultrasound in Congenital Segmental Giant Megaureter: A Case Report
}

\author{
Young Hun Jeon (ib ${ }^{1}$, Yu Jin Kim (iD ${ }^{1,}{ }^{*}$, Seok Joong Yun ${ }^{2}$, Bum Sang Cho (ii) ${ }^{3}$ and Ok Jun Lee ${ }^{4}$ \\ ${ }^{1}$ Department of Radiology, Chungbuk National University Hospital, Cheongju, Korea \\ ${ }^{2}$ Department of Urology, Chungbuk National University College of Medicine, Cheongju, Korea \\ ${ }^{3}$ Department of Radiology, Chungbuk National University College of Medicine, Cheongju, Korea \\ ${ }^{4}$ Department of Pathology, Chungbuk National University College of Medicine, Cheongju, Korea \\ "Corresponding author: Department of Radiology, Chungbuk National University Hospital, Cheongju, Korea. Tel: +82-432696503, Email: youkio97@naver.com
}

Received 2020 February 17; Revised 2020 March 04; Accepted 2020 April 25.

\begin{abstract}
Congenital segmental giant megaureter (CSGM), which refers to segmental cystic dilatation of the upper collecting system, is extremely rare in the pediatric population. Depending on the etiology, the condition could be categorized as primary or secondary megaureter with three subtypes including refluxing, obstructed, nonrefluxing and nonobstructed types. We report a case in which the CSGM presented as a giant abdominal cystic mass with associated right-sided hydronephrosis that was diagnosed by abdominal ultrasound. Cystic mass and the tubular structure at the caudal side of the cystic mass were demonstrated on abdominal ultrasound. We identified a connection between the cystic mass and the lumen of the tubular structure. Therefore, the cystic mass was reported as CSGM on abdominal ultrasound, especially categorized as the nonrefluxing and nonobstructed type. The percutaneous nephrostomy was performed to relieve hydronephrosis, followed by excision of the dilated segment. CSGM is an extremely rare presentation of megaureter, requiring variable imaging modalities and an appropriate intervention. Especially, careful real-time ultrasound was critical to identify the CSGM promptly and essential for the individual appropriate management.
\end{abstract}

Keywords: Hydronephrosis/Diagnostic Imaging, Infant, Urogenital Abnormalities/Surgery, Ureteral Obstruction/Congenital

\section{Introduction}

Megaureter is a comprehensive term for an expanded ureter that does not function normally, and it may be accompanied by dilatation of the upper collecting system. Especially segmental cystic dilatation of the ureter can be very rarely observed in cases of megaureter (1). Here, we present a rare case involving a neonate who exhibited a cystic abdominal mass on an antenatal ultrasound and was diagnosed with congenital segmental giant megaureter (CSGM) on the basis of ultrasound, voiding cystourethrography (VCUG), computed tomography (CT), and surgery.

\section{Case Presentation}

A one-day-old girl was admitted for evaluation of a large abdominal cystic mass and right-sided hydroureteronephrosis (Onen's grade III) (2) that was discovered during an antenatal ultrasound examination at another hospital. An abdominal ultrasound (IU22 Intelligent Ultrasound System; Philips, USA; $12-5-\mathrm{MHz}$ linear transducer) revealed a well-circumscribed cystic mass measuring $6.2 \times 6.7 \times 3.2 \mathrm{~cm}$ with multi-layered wall in the right lower abdomen (Figure 1A). Ipsilateral renal pelvocalyceal dilatation and calyceal blunting was observed with increased renal parenchymal echogenicity (Figure 1B). Sonography of the contralateral kidney showed normal parenchymal echogenicity with preserved corticomedullary differentiation. The patient also retained stable renal function (serum creatinine, 0.71 $\mathrm{mg} / \mathrm{dL}$ ). A small tubular cystic structure with peristalsis was observed between the cystic mass and the bladder. Continuity between the small tubular cystic structure and the cystic mass was noted during peristalsis of the small tubular cystic structure (Appendix 1 in Supplementary File), and the connection site had abrupt luminal narrowing. The diameter of the small tubular cystic structure did not exceed $0.5 \mathrm{~cm}$ and the length was measured as $1.2 \mathrm{~cm}$ (Figure 1C).

Therefore, the authors reported the cystic mass as a CSGM, and the small tubular cystic structure was considered a normal distal ureter. After 3 days of birth, contrastenhanced dynamic CT was performed for robust convincement. A CT scanner (SOMATOM Definition Flash; Siemens) 

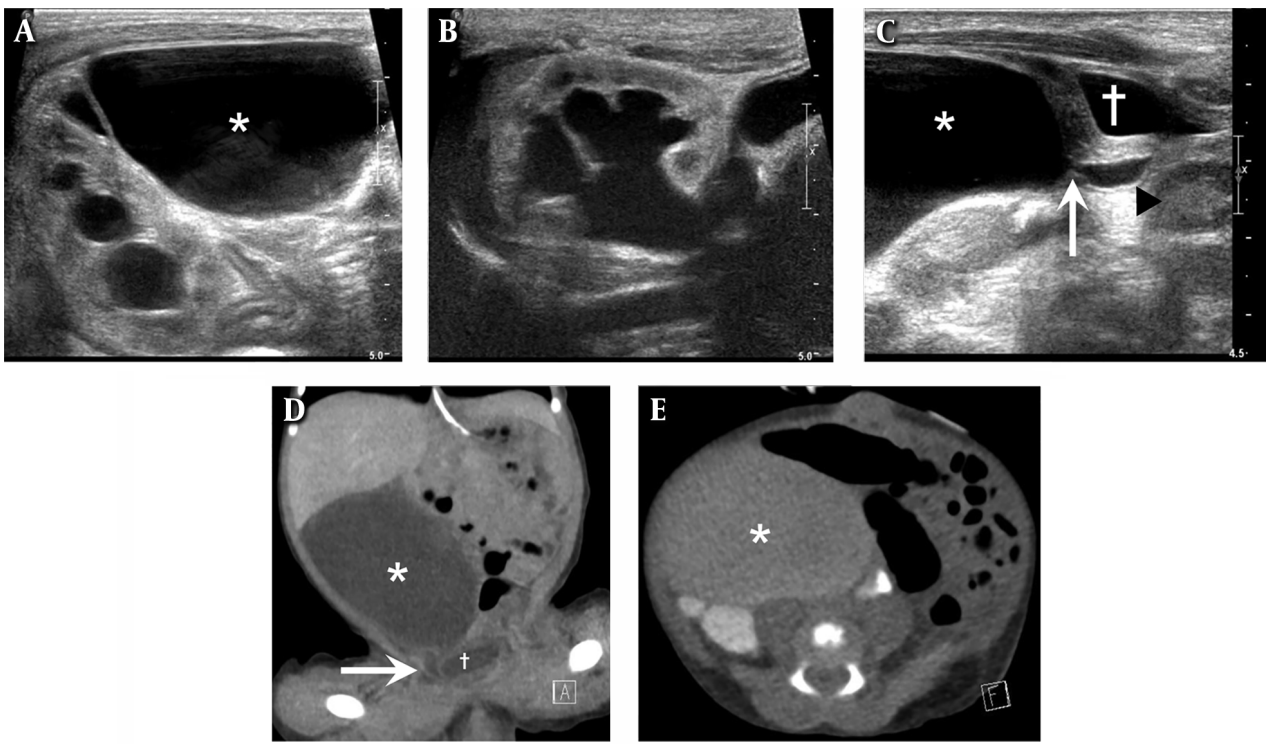

Figure 1. A one-day-old girl with a large cystic abdominal mass and right-sided hydroureteronephrosis discovered during an antenatal ultrasound examination. Abdomina ultrasound and abdominal contrast-enhanced CT. A, Axial ultrasonography (US) image shows a large cystic mass (asterisk) with multi-layered wall in the right upper abdomen; B, Ultrasound shows right pelvocalyceal dilatation and calyceal blunting with increased renal parenchymal echogenicity; C, Ultrasound identifies the continuity between the cystic mass (asterisk) and the cystic tubular structure (arrowhead). Abrupt luminal narrowing (white arrow) at the connection site is noted. The cystic tubular structure is connected with the urinary bladder (cross) with normal peristalsis. Peristalsis of the small cystic tubular structure is presented on video 1; D, Coronal view of contrastenhanced CT scan demonstrates the cystic mass (asterisk) in the right lower abdomen and small cystic tubular structure (white arrow) which is between the cystic mass and urinary bladder (cross); E, Axial view of 2-hour delayed CT scan shows opacification of the urinary system and the cystic mass (asterisk) with excreted contrast.

was used with low dose protocol. Delayed CT scans were performed to ensure that the cystic mass which presumed to be a CSGM and bladder were opacified, and were reduced the scan range as $3 \mathrm{~cm}$ to decrease the radiation dose. The well-circumscribed cystic mass was located at right lower quadrant area and the maximal diameter was measured as $6 \mathrm{~cm}$. A $1.2 \mathrm{~cm}$ long cystic tubular structure between the cystic mass and the bladder was also identified as demonstrated at ultrasonography (US) (Figure1D). The cystic mass and bladder were simultaneously opacified in a 2-hour delayed CT scan (Figure 1E), and the diagnosis of a CSGM was reconfirmed by contrast enhanced CT.

Decompression of hydronephrosis was considered necessary for preservation of the right kidney function. Accordingly, ultrasound-guided percutaneous 6-Fr pigtail catheter insertion was performed at 6 days after birth. Tubography revealed abrupt luminal changes at the proximal and distal ends of the CSGM. No other structural abnormalities were presented at tubography. Subsequent VCUG revealed negative findings.

Collectively, the examinations indicated the absence of vesicoureteral reflux or any obstructive causes; therefore, the patient was diagnosed as nonrefluxing and nonobstructed CSGM.

At 16 days after birth, the patient underwent surgery to excise the dilated segment with right ureteroureterostomy. Intraoperative findings confirmed severe segmental dilatation of the right ureter at approximately $3 \mathrm{~cm}$ proximal to the right ureterovesical junction (Figure 2A).

In the histopathological examination of the excised ureter, the nondilated segment showed normal distribution of smooth muscle tissue below the urothelial epithelium and a normal ratio of muscular tissue to fibrous tissue. In the dilated segment, the smooth muscle tissue layer was replaced by abundant connective tissue cells and vascular elements. This indicated structural changes in the smooth muscle fibers with replacement by a fibrous tissue layer. (Figure $2 \mathrm{~B}$ and $\mathrm{C}$ ).

A dimercaptosuccinic acid renal scan performed at 3 months after surgery showed no cortical defects, while an ultrasound performed at 9 months showed normal size and parenchymal echo of the right kidney with no dilatation of the renal collecting system and ureter. The patient was observed to be asymptomatic during 18 months of follow-up period, furthermore at the last abdominal ultrasound, both kidneys had normal parenchymal echogenicity with preserved corticomedullary differentiation and right-sided hydronephrosis was resolved. 

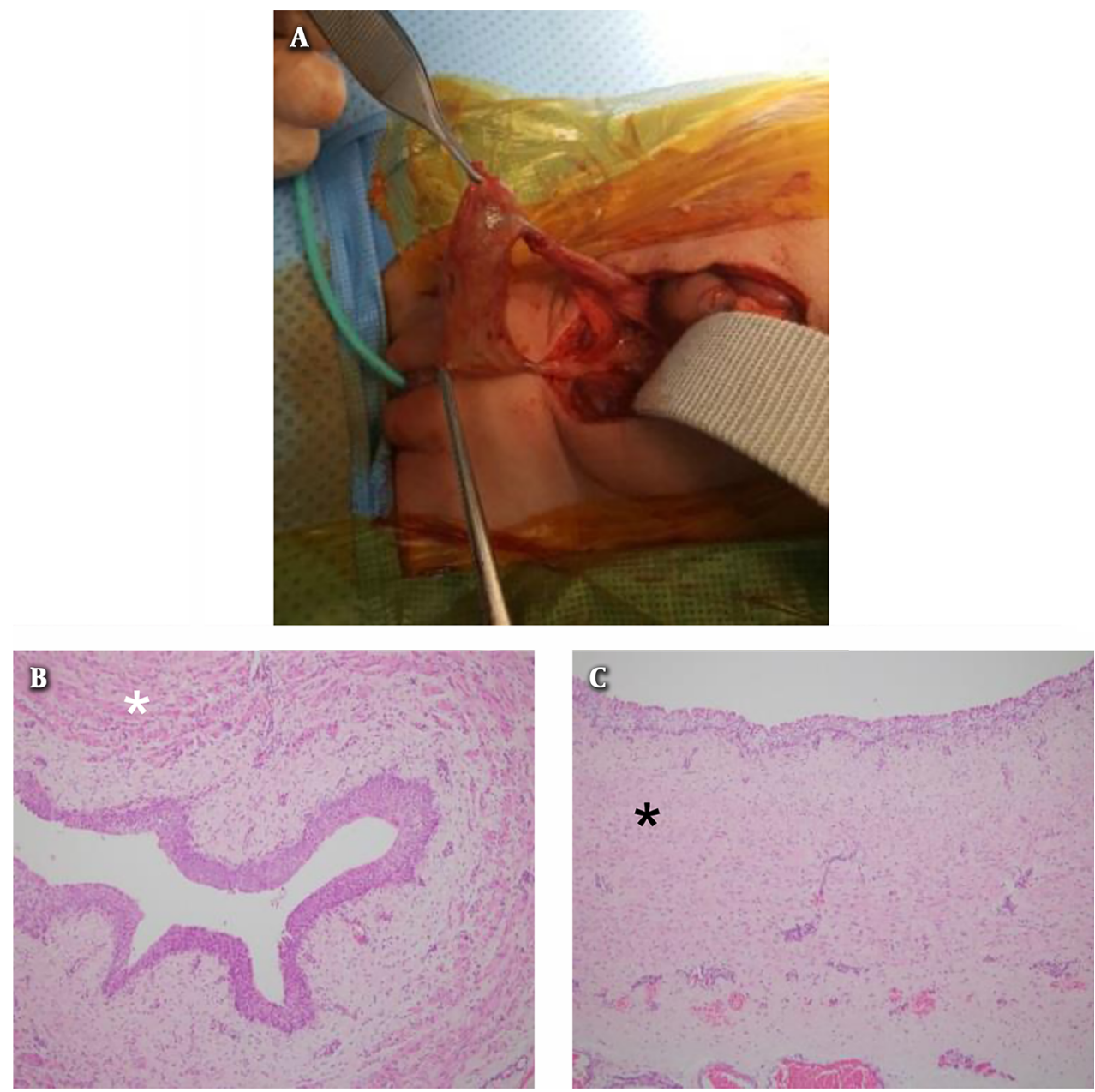

Figure 2. Intraoperative photograph and photomicrograph of the specimen. A, Intraoperative photograph shows the dilated segment of the right ureter of the congenital segmental giant megaureter; B and C, Photomicrograph of the cross-sectional view of the non-dilated segment; B, Exhibits normal distribution of the smooth muscle tissue (white asterisk) and photomicrograph of the cross-sectional view of the dilated segment; C, Shows abundant vascular elements and connective tissue under the urothelial epithelium, with marked dissociation of smooth muscle bundles (black asterisk) (hematoxylin and eosin, $100 \times$ ).

\section{Discussion}

In children, the congenital megaureter is defined as congenital condition with diffuse or segmental dilatation of the lumen of a ureter 10 times more than normal diameter of ureter ( $\leq 5 \mathrm{~mm}$ ) (3). The condition can be categorized as primary or secondary megaureter, and depending on the etiology, it can be divided into refluxing, obstructed, nonrefluxing and nonobstructed types (4). There are few published reports on the pathogenesis of the congenital megaureter, and the mechanisms can be explained by poor development of longitudinal muscle (5). Decreased ratio of muscular tissue to connective tissue leads to lack of coordination between muscular tissue and connective tissue at the distal ureter, resulting in a decrease or the loss of ureteral motility (6).

Up to our knowledge, there were less than 10 published case reports in the literature since the first congenital giant megaureter was reported in 1964 (7). Especially, CSGM is extremely rare and only 5 published case reports since Ramaswamy et al. (8) reported first case of CSGM in 1995. Goto et al. (9) revealed a case of CSGM from birth with use of MRI. In addition, there were other three case reports of CSGM with or without ectopic kidney or renal hypoplasia, and the patients were over 6 years old on diagnosis with use of CT or without preoperative imaging (10-12). In contrary to published reports, we initially diagnosed CSGM immediately after delivery at abdominal ultrasound and revealed the first case report of successful preoperative diagnosis of CSGM by fully use of ultrasound, which is primary 
imaging modality for neonate and enable to real-time diagnosis.

For infants and children, ultrasound is a primary imaging examination for the kidneys and ureters without radiation. Ultrasound can be helpful for differentiation of CSGM from other abdominal cystic masses found in infants such as duplication cyst or cystic teratoma, and mesenteric cyst. In our case, the cystic abdominal mass had continuity from renal collecting system with layered wall and peristalsis on US, therefore other differential diagnosis was excluded. In addition, ureteral peristalsis can be identified on the basis of real-time and careful ultrasound examination as the present case.

With regard to the treatment of CSGM, conservative management or surgical treatment can be considered depending on the circumstances. Important factors that need consideration during treatment planning include the presence or absence of accompanying deformities, ipsilateral renal function, and the length of the ureteral segment showing normal function. Generally, surgical treatment involving dilated segment excision, ureteral reimplantation, or ureteroureterostomy is effective in treating CSGM while preserving renal function. However, ipsilateral nephroureterectomy should be performed in cases with abnormal function of the ipsilateral ureter and/or kidney or accompanying deformities.

In conclusion, we presented a rare case of CSGM presenting as a cystic abdominal mass in a neonate. This is an extremely rare presentation of megaureter, and prompt and accurate diagnosis in our case was possible by the use of different serial imaging modalities and an appropriate intervention. Especially, the real-time ultrasound is helpful to identify the CSGM promptly and essential to manage based on patients' specific condition.

\section{Supplementary Material}

Supplementary material(s) is available here [To read supplementary materials, please refer to the journal website and open PDF/HTML].

\section{Footnotes}

Authors' Contributions: Study concept and design: YJK. Analysis and interpretation of data: YHJ and YJK. Drafting of the manuscript: YHJ. Critical revision of the manuscript for important intellectual content: YJK and YHJ. Administrative, technical, and material support: SJY, OJL, and BSC. Study supervision: YJK.

Conflict of Interests: None of the authors have any potential conflicts to disclose (financial, professional, or personal) that are relevant to the manuscript.
Ethical Approval: This retrospective study was approved by the Institutional Review Board at our institution. The ethical approval was waived by the Institutional Review Board due to its retrospective nature.

Funding/Support: The authors received no funding or support in connection with this study.

\section{References}

1. Yu M, Ma G, Ge Z, Lu R, Deng Y, Guo Y. Unilateral congenital giant megaureter with renal dysplasia compressing contralateral ureter and causing bilateral hydronephrosis: A case report and literature review. BMC Urol. 2016;16:7. doi: 10.1186/s12894-016-0125-y. [PubMed: 26860315]. [PubMed Central: PMC4746910].

2. Onen A. An alternative grading system to refine the criteria for severity of hydronephrosis and optimal treatment guidelines in neonates with primary UPJ-type hydronephrosis.JPediatr Urol.2007;3(3):200-5. doi: 10.1016/j.jpurol.2006.08.002. [PubMed: 18947735].

3. Berrocal T, Lopez-Pereira P, Arjonilla A, Gutierrez J. Anomalies of the distal ureter, bladder, and urethra in children: embryologic, radiologic, and pathologic features. Radiographics. 2002;22(5):1139-64. doi: 10.1148/radiographics.22.5.g02se101139. [PubMed: 12235344].

4. Lockhart JL, Singer AM, Glenn JF. Congenital megaureter. J Urol. 1979;122(3):310-4. doi: 10.1016/s0022-5347(17)56380-6.

5. Mackinnon KJ, Foote JW, Wiglesworth FW, Blennerhassett JB. The pathology of the adynamic distal ureteral segment. J Urol. 1970;103(2):134-7. doi: 10.1016/s0022-5347(17)61908-6. [PubMed: 5410587].

6. Vlad M, Ionescu N, Ispas AT, Ungureanu E, Stoica C. Morphological study of congenital megaureter. Rom J Morphol Embryol. 2007;48(4):381-90. [PubMed: 18060188].

7. Chatterjee SK. Giant megaureter. Br J Urol. 1964;36:406-12. doi: 10.1111/j.1464-410x.1964.tb09527.x. [PubMed:14209039].

8. Ramaswamy S, Bhatnagar V, Mitra DK, Gupta AK. Congenital segmental giant megaureter. J Pediatr Surg. 1995;30(1):123-4. doi: 10.1016/00223468(95)90629-0. [PubMed: 7722817].

9. Goto H, Kanematsu A, Yoshimura K, Miyazaki Y, Koyama T, Yorifuji T, et al. Preoperative diagnosis of congenital segmental giant megaureter presenting as a fetal abdominal mass. J Pediatr Surg. 2010;45(1):269-71. doi: 10.1016/j.jpedsurg.2009.09.031. [PubMed: 20105619].

10. Paonam S, Bag S. Congenital Segmental Giant Megaureter Presenting as Cystic Abdominal Mass in a Child. J Clin Case Rep. 2015;5(10). doi: 10.4172/2165-7920.1000614.

11. Khanna A, Mavuduru RS, Bora GS, Devana SK, Mandal AK. Congenital segmental megaureter in an ectopic kidney. Urology. 2018;113:e7-8. doi:10.1016/j.urology.2017.11.032. [PubMed: 29203188].

12. Pinter AB, Szabo L, Szever ZS, Magyarlaki T. Bilateral congenital seg mental megaureter. J Urol. 1997;158(2):570-1. [PubMed: 9224363]. 